\title{
Pinching Dynamics and Satellite Droplet Formation in Symmetrical Droplet Collisions
}

\author{
Kuan-Ling Huang, ${ }^{1}$ Kuo-Long Pan $\odot,{ }^{1, *}$ and Christophe Josserand ${ }^{2}$ \\ ${ }^{1}$ Department of Mechanical Engineering, National Taiwan University, Taipei 10617, Taiwan, Republic of China \\ ${ }^{2}$ LadHyX, CNRS and Ecole Polytechnique, UMR 7646, IP Paris, 91128 Palaiseau, France
}

(Received 1 May 2019; published 6 December 2019)

\begin{abstract}
In head-on collisions between two droplets, reflexive separation is frequently formed, showing tentative coalescence followed by disintegration into two primary drops. With higher impact inertia relative to surface tension, characterized by a Weber number (We), more satellite droplets are created between the primary drops. In the symmetric configuration, the existing phenomenological models indicate the absence of satellite droplets at the onset We when the coalesced drops start to break. Supported by experimental and simulation evidence, here we demonstrate the exclusive formation of at least one droplet after pinch of the thread connecting the colliding drops. In accordance with the universal features of a thinning liquid filament approaching singularity as predicted by scaling theories of pinch-off, the mechanism of satellite droplet formation in the symmetrical impact of droplets is clarified. Via slight breaking of the symmetry, no satellite droplet can be observed, thus providing a possible interpretation for the discrepancy in the literature and implications for controlling undesirable drop formation.
\end{abstract}

DOI: 10.1103/PhysRevLett.123.234502

Droplet collision plays a critical role in classical fluid mechanical research not only for the fundamental significance but also for its high relevance to many natural disciplines and practical processes. Examples are seen in raindrop formation [1,2], combustion in spray engines [3,4], operation of nuclear reactors $[5,6]$, and medical treatments such as inhalation therapy $[7,8]$. For mastering the mass distribution of liquid in the targeted systems, the collision dynamics of two droplets have been studied extensively in recent decades [9-15]. Regarding the symmetrical collision of droplets, reflexive separation (RS) occurs when the impact inertia is large enough to overcome the surface tension force, as indicated by the Weber number, We $=$ $2 \rho U^{2} R / \sigma$. Here $U$ is the relative velocity of two droplets, $R$ the radius, and $\rho$ and $\sigma$, respectively, the density and surface tension of the liquid. RS features a strong internal flow with a rebounding motion, which elongates the coalesced drop and leads to a dumbbell shape followed by fragmentation. As shown in Ref. [9], collisions of near-inviscid (water) droplets at the critical $\mathrm{We}\left(\mathrm{We}_{c}\right)$ at the onset of RS led to the formation of only two drops after rupturing, with nearly identical sizes. For slightly viscous drops (hydrocarbons), as studied in Ref. [10,11], however, a satellite droplet with a much smaller size was observed along with two primary drops when the We slightly exceeded $\mathrm{We}_{c}$. Despite the discrepant results for various Ohnesorge numbers (Oh $=\mu / \sqrt{\rho R \sigma}$, where $\mu$ is the fluid viscosity) of droplet collisions upon the onset of RS, phenomenological models were proposed along the same lines. That is, they all indicated the occurrence of pinch-off right at the center of the ligament connecting the primary drops, without any satellite droplet produced afterward [9,11-13].
On the other hand, satellite droplets were observed in the breakup of Newtonian liquid filaments for a wide range of Oh (0.0018-1.81) [16-18]. The formation of satellite droplets was attributed to the asymmetrical singularity of pinch-off [19-22]. To depict the dynamics of the thinning liquid filaments, scaling theories of inertial $(I)$ [23], viscous $(V)$ [24], and inertial-viscous (IV) [19] regimes have been developed. While inertial and capillary forces balance in the $I$ regime, viscous and capillary forces balance in the $V$ regime, and all three forces (inertial, viscous, and capillary) balance in the $I V$ regime. A recent study [18] demonstrated that, for a liquid filament with slightly viscous $(\mathrm{Oh}=0.07)$ and highly viscous liquids $(\mathrm{Oh}>1.0)$, the $I V$ regime dominated the last stage of pinching. For nearly inviscid liquid, however, the experiments and simulations showed that the thinning liquid filament followed the $I$ regime when it finally approached singularity $[25,26]$. This scenario remained valid even when Oh was up to 0.02 [27]. In fact, depending on the $\mathrm{Oh}$ and also the initial conditions, the observed dynamics can alternate between the different regimes $(I, V$, and $I V)$ [18,28]. To correlate with the pinching processes and resolve the puzzle regarding the creation of satellite drops, in this Letter, the breakup of RS in a considerable range of Oh (0.0061-0.152) was experimentally studied for a We close to $\mathrm{We}_{c}$ (within $\pm 15 \%$ ). Furthermore, full-field numerical simulations have been performed to understand the pinching dynamics during the head-on droplet impact.

To identify the generic behaviors of RS for a wide range of Oh, drops of water, alkanes, and silicone oils with different diameters have been tested. Collisions of two identical droplets with the desired We and Oh were carried 


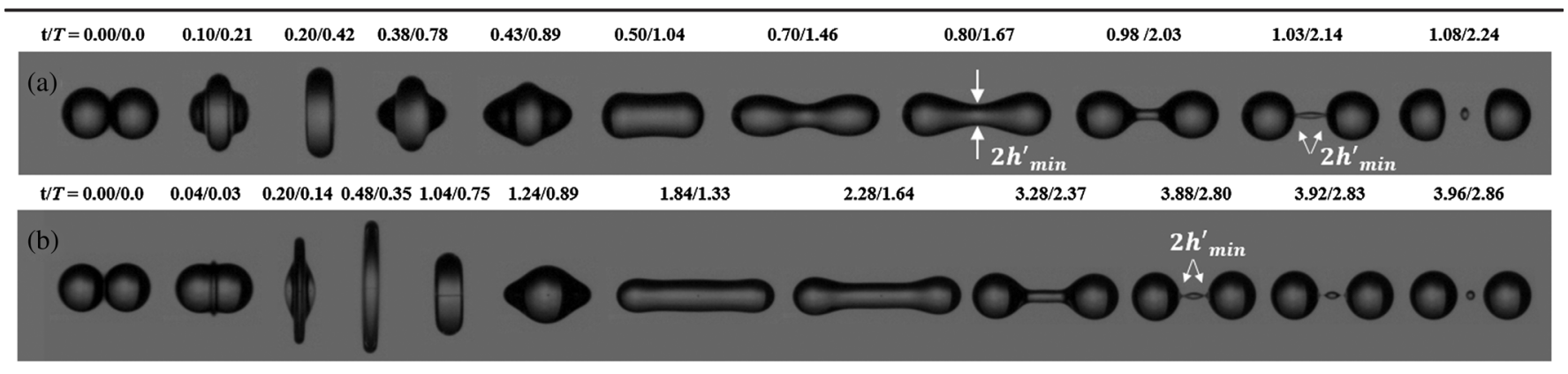

FIG. 1. Collision sequences of (a) two water droplets with $D=2 R=0.3 \mathrm{~mm}$, We $=20.0$, and Oh $=0.0089$, and (b) silicone oil droplets with $D=0.398 \mathrm{~mm}, \mathrm{We}=197.3$, and $\mathrm{Oh}=0.152$. The time unit of $t$ is a millisecond, and that of $T$ is the natural oscillation time $(\pi / 4) \sqrt{\rho D^{3} / \sigma}$.

out by a conventional drop-on-demand method using piezoelectric plates. The impact events were recorded by a high-speed camera with 20000-25000 frames/s, and the spatial resolution was up to 188 pixels $/ \mathrm{mm}$ by using a microscope lens. Moreover, another CCD camera was set on top to ensure that all collisions happened on the same focal plane. More details are provided in the Supplemental Material [29].

The evolution of RS for a near-inviscid liquid (water, $\mathrm{Oh}=0.0089$ ) is shown in Fig. 1(a). After impact, the coalesced drop is flattened $(t=0.0-0.2 \mathrm{~ms})$ and then reflexes to a cylinder with two rounded ends $(0.2-0.5 \mathrm{~ms})$. After reaching the maximum length $(0.8 \mathrm{~ms})$, the elongated drop develops to a dumbbell shape $(0.8-0.975 \mathrm{~ms})$. This is followed by a shift of the location showing the minimum radius $\left(h_{\min }^{\prime}\right)$ from the center toward the outer bulbs $(0.975-$ $1.025 \mathrm{~ms})$, leading to the formation of two asymmetrical necks and, consequently, a satellite droplet (1.075 ms). The cone angle $(\theta)$ of the neck as defined in Fig. 2 is about $18.0^{\circ}$, showing great agreement with the theoretically derived [19] and experimentally observed [32] quantities of a thinning liquid filament before pinch-off.

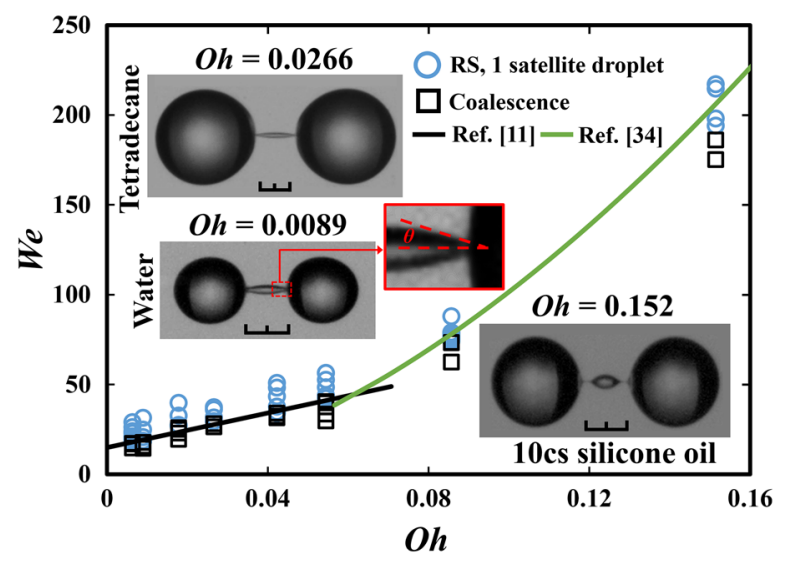

FIG. 2. The boundary between coalescence and RS on the WeOh diagram. (Insets) Experimental profiles of the drop before breakup. Scaler bar, $200 \mu \mathrm{m}$.
For a more viscous liquid (silicone oil, $\mathrm{Oh}=0.152$ ), as shown in Fig. 1(b), the same scenario is observed, ending in the formation of a satellite droplet. Before breakup, the two asymmetrical necks pinch to microthreads (3.92 ms), which can be seen as well in the pinch-off of a dripping drop made of slightly and highly viscous liquids [18,27,33]. The transition from the dumbbell-like drop with a center neck to that with double necks at opposite locations always occurred at the relaxation stage of the separation process. Figure 2 shows the boundary between coalescence and RS in a We-Oh phase diagram. At least one satellite droplet is produced when rupture occurs. Furthermore, the transition boundary, $\mathrm{We}_{c}(\mathrm{Oh})$, follows the predictions of theoretical models for binary droplet separation with low [11] and high Oh values [34], respectively. Since the initial coalescence process is not universal and is influenced in particular by the ambient pressure or drop oscillations, this boundary is sensitive to external conditions [11,35]. This might explain the small overlap of behaviors near the boundary as well as the discrepancy between the theoretical predictions and the experiments. The experimental observation-that a merged drop associated with a center neck develops to a drop with two mirrored necks, leading to the formation of a satellite droplet-is consistent with the evolution of a pinching liquid filament [16-18,36,37]. This reveals that the underlying mechanism for the fragmentation caused by a symmetrical droplet collision surrenders a similar physics to the pinching free-surface flow.

To track the flow structure causing pinch-off of RS, the open-source numerical code GERRIS $[38,39]$ has been used to simulate the breakup process of coalesced drops. The finite volume and volume-of-fluid methods are used to solve the axisymmetric incompressible Navier-Stokes equations and track liquid-gas interfaces, respectively. In addition, the quad or octree discretization is conducted to resolve the thin necks of coalesced drops, whose width evolves in a large range of length scales [40,41]. It was observed via a numerical simulation that the final regime of the pinching process occurred when the dimensionless minimum radius, $h_{\min }=h_{\min }^{\prime} / R$, was about an order of 

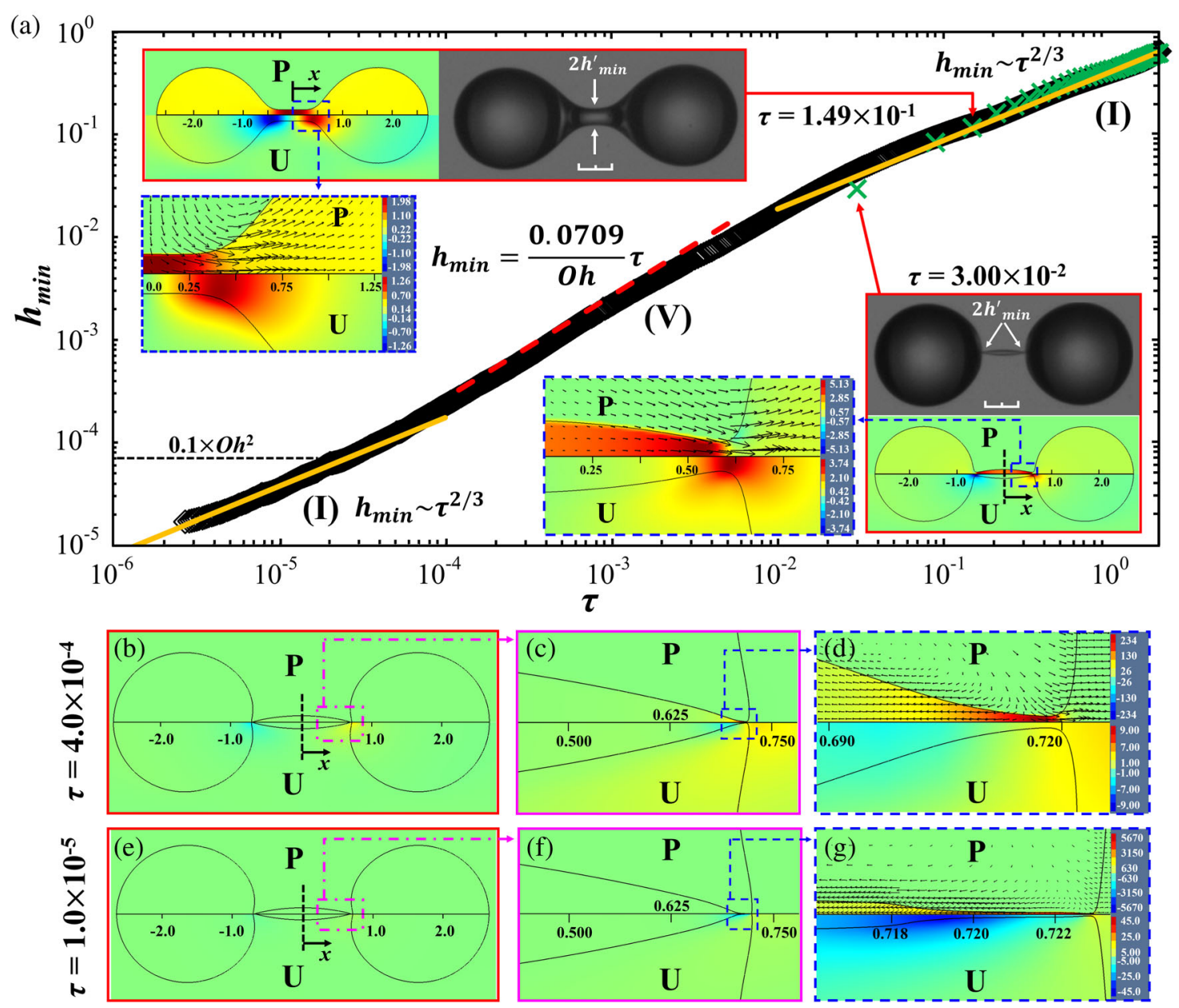

FIG. 3. (a) Experimental snapshots and numerical simulations of the distributions of pressure and axial velocity for Oh $=0.0266$ and $\mathrm{We}=29.4$, as well as the variation of $h_{\min }$ with $\tau$. Yellow solid line, $I$ regime; red dashed line, $V$ regime; green cross, experimental values of $h_{\min }$; black open diamond, simulation. Scaler bars in the experimental images, $200 \mu \mathrm{m}$. (b) The drop profile, pressure, and axial velocity fields at $\tau=4.0 \times 10^{-4}$. (c) The interface of the outer primary drop attached to the thinning neck is overturned. (d) Distributions of pressure and velocity vectors around the asymmetrical neck at $\tau=4.0 \times 10^{-4}$. (e) The drop profile, pressure, and axial velocity fields at $\tau=1.0 \times 10^{-5}$, showing (f) the overturned interface of the outer primary drop, and (g) the microthread produced by the reversed flow.

magnitude smaller than $\mathrm{Oh}^{2}$ [18]. Therefore, the minimum grid sizes in the present simulations, which were set as small as $\sim 0.01 \mathrm{Oh}^{2}$, were $5 \mathrm{~nm}\left(R / 2^{16}\right)$ and $49 \mathrm{~nm}\left(R / 2^{12}\right)$ for the cases of $\mathrm{Oh}=0.0266$ and 0.152 , respectively. As shown in Figs. 3 and 4, the simulations accurately reproduced the geometrical features of deformed drops in the experiments. Furthermore, the computed variations of dimensionless $h_{\min }$ with $\tau$ agree well with the experimental measurements, where $\tau=\left(t_{b}-t\right) \sqrt{\sigma / \rho R^{3}}$, and where $t_{b}$ is the time of the breakup. More numerical details are given in the Supplemental Material [29].

Figure 3(a) shows the variation of $h_{\min }$ with $\tau$ as well as the transient pressure and axial velocity fields for the collision of droplets with $\mathrm{Oh}=0.0266$ and $\mathrm{We}=29.42$. During the thinning process, the neck passes through the $I$ regime, the $V$ regime, and finally the $I$ regime before breakup. In the $I$ regime, the variation of $h_{\min }$ follows the scaling of $h_{\min } \sim \tau^{2 / 3}$ [23], and $h_{\min } \approx 0.0709 \tau / \mathrm{Oh}$ for the $V$ regime [24]. At the earlier $I$ regime, the fluids are stagnant at the drop center $(x=0)$ with the maximum pressure [the Fig. 3(a) insets of $\tau=1.49 \times 10^{-1}$ ], which drives the flow from the neck to the outer bulbs of the drop, making the maximum axial velocities locate at $x= \pm 0.42$. This makes the local radius along the drop decrease fastest at $x \approx \pm 0.54$ and leads to shifts in the locations of maximum pressure, and of $h_{\min }$ from $x=0$ to $x \approx \pm 0.54$ and $x \approx \pm 0.59$ [the Fig. 3(a) insets of $\left.\tau=3.0 \times 10^{-2}\right]$, respectively. As the necks keep thinning from the $I$ to $V$ regimes $\left(\tau=4.0 \times 10^{-4}\right)$, a thread at the center is developed into an island, as shown in Fig. 3 (b), and the connecting interfaces of the outer bulbs are overturned [Fig. 3(c)]. At this stage, the locations of the maximum pressure and $h_{\text {min }}$ move to $x \approx \pm 0.72$, whereby a stagnation zone is formed, as shown in Fig. 3(d). The high capillary pressure at this stagnation zone reverses the flow 


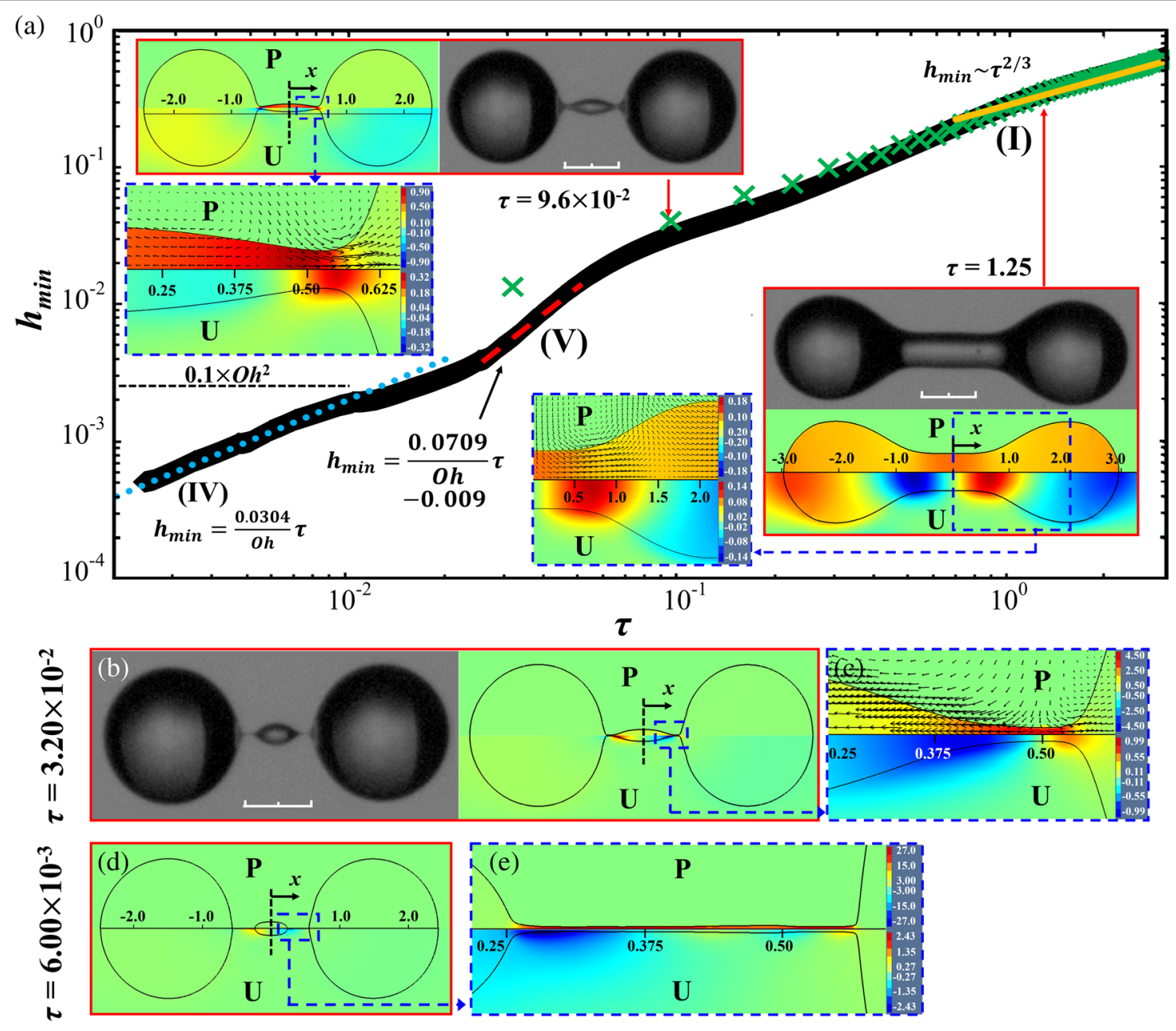

FIG. 4. (a) Experimental snapshots and numerical simulations of the distributions of pressure and axial velocity for Oh $=0.152$ and $\mathrm{We}=197.3$, as well as the variation of $h_{\min }$ with $\tau$. Yellow solid line, $I$ regime; red dashed line, $V$ regime; blue dotted line, $I V$ regime; green cross, experimental values of $h_{\min }$; black open diamond, simulation. Scaler bars in the experimental images, $200 \mu \mathrm{m}$. (b) Comparison between the experimental image and the calculated drop profile at $\tau=3.2 \times 10^{-2}$. (c) Distributions of velocity vectors and pressure around the neck at $\tau=3.2 \times 10^{-2}$. (d) The drop profile, pressure, and axial velocity fields at $\tau=6.0 \times 10^{-3}$, showing (e) the microthread with a length of about $60 \mu \mathrm{m}$.

toward the island and simultaneously forces the fluids out of the asymmetrical necks into the outer bulbs. Consequently, the variation of decreasing $h_{\min }$ at the last stage when $h_{\min } \sim 0.1 \mathrm{Oh}^{2}$ exhibits the scaling law of the $I$ regime, as shown in Fig. 3(a). This result is consistent with the previous experimental observation of a pinching liquid bridge, indicating that the pinching dynamics at the final stage for a near-inviscid filament follows the $I$ regime [2527,32]. In addition, as shown in Figs. 3(f) and 3(g), the asymmetrical neck was narrowed to a thread with a length of about $9 \mu \mathrm{m}$ and a radius of about $33 \mathrm{~nm}$ by the reversed flow close to the stagnation point ( $I$ regime, $\tau=1.0 \times 10^{-5}$ ).

For more viscous droplets $(\mathrm{Oh}=0.152$ and $\mathrm{We}=197.3)$, Fig. 4(a) shows that the thinning necks of the united drop sequentially undergo the $I$ regime, the $V$ regime, and, eventually, the $I V$ regime. From $\tau=1.25$ ( $I$ regime) to $\tau=$ $9.6 \times 10^{-2}$ ( $V$ regime $)$, the shifts in the maximum pressure and $h_{\min }$ from $x=0$ to $x \approx \pm 0.54$ can be observed in the insets with blue-dashed borders in Fig. 4(a). Unlike the less viscous case of $\mathrm{Oh}=0.0266$, the stagnation zones have already been built halfway $\left(\tau=9.6 \times 10^{-2}\right)$ between the $I$ and $V$ regimes. This contributes to the formation of two microthreads with a length:radius ratio of about $22 / 1.6 \mu \mathrm{m}$ at $\tau=3.2 \times 10^{-2}$ ( $V$ regime) which connect the island and the outer bulbs, as shown in Figs. 4(b) and 4(c). Finally each of the elongating filaments develops to a shape with length: radius ratio of about $60 / 1.2 \mu \mathrm{m}$ at $\tau=6.0 \times 10^{-3}$ [Figs. 4 (d) and 4(e) $]$ and the stage enters the $I V$ regime, showing $h_{\min }=$ $0.0304 \tau / \mathrm{Oh}[19]$.

The simulations in Figs. 3 and 4 demonstrate that after the neck is formed at the center, the fluids accelerate outward between the regions of high and low pressure, i.e., the regions around the flatter interface at the center and the steeper profiles next to the two outer bulbs, respectively. 


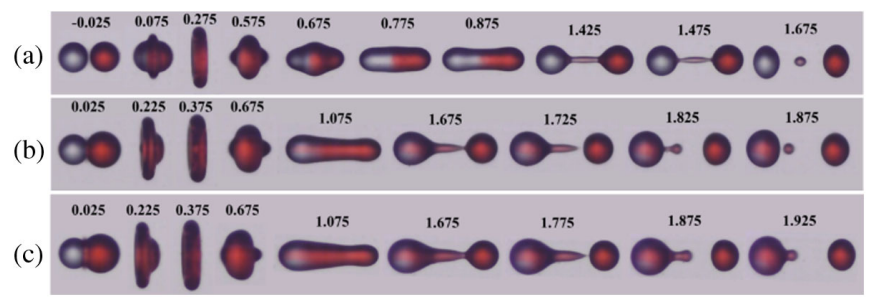

FIG. 5. The collision sequences of tetradecane droplets with (a) $A=1.0, U=2.29 \mathrm{~m} / \mathrm{s}$; (b) $A=1.17, U=2.26 \mathrm{~m} / \mathrm{s}$; and (c) $A=1.28, U=2.30 \mathrm{~m} / \mathrm{s}$. Here, for the smaller droplet, $R_{s}=$ $150 \mu \mathrm{m}$ and $\mathrm{Oh}_{s}=0.0376$. Time unit, $\mathrm{ms}$.

With nearly stagnant fluids around the center, the elongated drop thins fastest at the mirrored necks, leading to formation of an island at the center which subsequently develops to a satellite droplet. Such a symmetrical scenario containing two asymmetric pinch-off's in RS is consistent with the generic breakup of contracting liquid filaments and film bridges $[18,20,22,23,41,42]$. Therefore, in accordance with the universality of the asymmetrical singularity and scaling laws in pinching free-surface flow [19-24,43], a symmetric breakup right at the center of tentatively united drops after head-on impact can never result. There must be one satellite droplet formed at the onset of RS.

To investigate possible ways to elimination of the satellite droplet, the symmetry of head-on collision is broken by slight change of the size ratio of impact droplets [44], which could also be achieved by other means such as using droplets of dissimilar liquid properties [13,45-47]. The effect of size ratio $\left(A=R_{l} / R_{s}\right)$ on the satellite droplet formation, where $R_{l}$ and $R_{s}$ are the radius of the large and small droplets, respectively, is demonstrated by the sequences in Fig. 5 for tetradecane droplets. With a symmetry [Fig. 5(a)], a satellite droplet is created and there is no mixing in the two primary drops after collision. When the size ratio is raised to 1.17, as shown in Fig. 5(b), some mass of the larger drop (dyed red) penetrates into the smaller one (transparent originally) and causes internal stirring at the relaxation stage $(t=1.075 \mathrm{~ms})$. The liquid from the originally larger droplet enlarges the smaller one and thickens the neck beside it $(1.675 \mathrm{~ms})$. While both necks taper as the drop elongates, the thinner neck breaks earlier
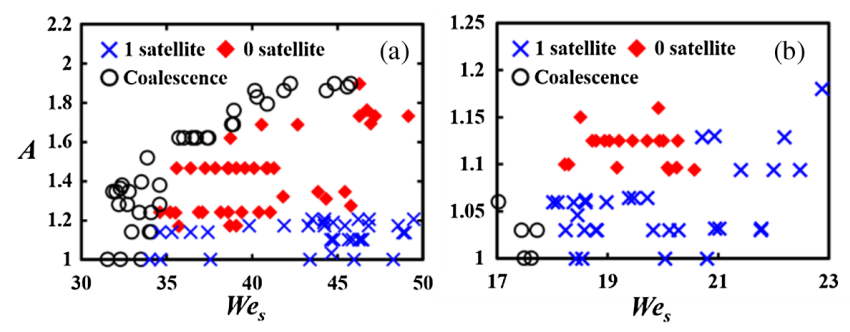

FIG. 6. Formation of satellite droplets in collisions of (a) tetradecane with $\mathrm{Oh}_{s}=0.0376$ and (b) water with $\mathrm{Oh}_{s}=0.0089$. $R_{s}=0.3 \mathrm{~mm}$. $(\sim 1.725 \mathrm{~ms})$. A round end of center mass is formed by the pinching point at $t=1.825 \mathrm{~ms}$ and recedes to the left. Then the thicker neck ruptures as well (1.875 ms), leading to formation of a satellite droplet. By further increasing $A$, substantial asymmetry is created after impact, as shown in Fig. 5(c). Consequently, after the thinner neck breaks $(1.775 \mathrm{~ms})$, the remaining mass of the thread is pulled more rapidly toward the left drop (now larger) by the surface force. Subsequently it merges with the left drop before a significant thinning of the thicker neck can be observed. Note that the dye additive, Sudan Red, made a negligible change on the fluid properties of tetradecane (within $0.4 \%$ ).

The variation of satellite droplet formation versus the size ratio is shown in Fig. 6, in terms of the We of the smaller droplet for two $\mathrm{Oh}_{s}$ values. For slightly viscous droplets (tetradecane, $\mathrm{Oh}_{s}=0.0376$ ), Fig. 6(a) shows that the satellite droplet can be eliminated when the difference in the radii of impinging droplets exceeds $18 \%$ of the smaller one. On the other hand, for the near-inviscid droplets (water, $\mathrm{Oh}_{s}=0.0089$ ), as shown in Fig. 6(b), only $10 \%$ of the size difference is needed to eliminate the satellite droplet.

In passing, we note that, though the symmetry of the droplet collision could be broken with a slight change of the impact angle, the experiment demonstrated very little effect on suppressing the satellite droplet. This was because RS was created within $12^{\circ}$ [29], and permanent coalescence occurred beyond this threshold. Similar results were also observed in the collisions of propanol droplets [12].

In summary, we have investigated the formation of satellite droplets near the threshold of RS in the symmetrical collision between two droplets for near-inviscid to slightly viscous fluids. The experiments have shown that in the wide range of Oh values (0.0061-0.152), at least one satellite droplet must be formed upon the onset of separation. Accordingly, the numerical simulations further demonstrate that the pinching necks of the temporarily coalesced drop, created at the two sides, are asymmetrical. The results agree well with the universal features of pinching free-surface flow and the scaling laws $[19,21,23,24]$. This demonstrates that pinch-off cannot occur in the middle between two primary drops [48]. That is, the profile nearing the breaking point is unlikely to be symmetrical, as was described in previous plots of reflexive separations $[9,11-13,45]$. The pinch-off induced by an asymmetrical singularity [21] is more likely to locate between a steeper free surface (primary drop on the outer side) and a flatter free surface (liquid thread on the inner side). Two pinched free surfaces with mirrored asymmetrical profiles thus permit the formation of a satellite droplet upon breakup. Moreover, the collisions between two droplets with unequal sizes demonstrate the effectiveness of asymmetrical impact on the suppression of satellite droplet formation. 
We thank the NTU Career Development Project (Grants No. NTU-CDP-106R7822, No. NTU-107L7822, and No. NTU-108L7822) for the funding support, and MOST-CNRS International Exchange Scheme and MOSTRS International Exchange Scheme for the mutual visits (Grants No. MOST-106-2911-I-002-503, No. MOST-1062911-I-002-541 and No. MOST-107-2911-I-002-524). We appreciate NCHC for the computing support. We are grateful for Professor J. T. Yang's support with a color high-speed camera.

*Corresponding author. panpeter@ntu.edu.tw

[1] R. Gunn, Science 150, 695 (1965).

[2] I. Strangeways, Precipitation: Theory, Measurement and Distribution (Cambridge University Press, Cambridge, England, 2006).

[3] H. H. Chiu, Prog. Energy Combust. Sci. 26, 381 (2000).

[4] D. Zhang, P. Zhang, Y. Yuan, and T. Zhang, Combust. Flame 173, 276 (2016).

[5] L. G. Moretto, K. Tso, N. Colonna, and G. J. Wozniak, Phys. Rev. Lett. 69, 1884 (1992).

[6] A. Menchaca-Rocha, M. Borunda, S. S. Hidalgo, F. Huidobro, K. Michaelian, and V. Rodriguez, Advances in Nuclear Dynamics 2 (Springer, Boston, 1996).

[7] K. R. May, J. Aerosol Sci. 4, 235 (1973).

[8] Y. Feng, C. Kleinstreuer, N. Castro, and A. J. Rostami, Aerosol Sci. Technol. 96, 96 (2016).

[9] N. Ashgriz and J. Y. Poo, J. Fluid Mech. 221, 183 (1990).

[10] Y. J. Jiang, A. Umemura, and C. K. Law, J. Fluid Mech. 234, 171 (1992).

[11] J. Qian and C. K. Law, J. Fluid Mech. 331, 59 (1997).

[12] G. Brenn, D. Valkovska, and K. D. Danov, Phys. Fluids 13, 2463 (2001).

[13] C. Planchette, E. Lorenceau, and G. Brenn, J. Fluid Mech. 702, 5 (2012).

[14] K. L. Pan, C. K. Law, and B. Zhou, J. Appl. Phys. 103, 064901 (2008).

[15] G. Brenn and V. Kolobaric, Phys. Fluids 18, 087101 (2006).

[16] B. Ambravaneswaran, E. D. Wilkes, and O. A. Basaran, Phys. Fluids 14, 2606 (2002).

[17] A. A. Castrejon-Pita, J. R. Castrejon-Pita, and I. M. Hutchings, Phys. Rev. Lett. 108, 074506 (2012).

[18] J. R. Castrejón-Pita, A. A. Castrejón-Pita, S. S. Thete, K. Sambath, I. M. Hutchings, J. Hinch, J. R. Lister, and O. A. Basaran, Proc. Natl. Acad. Sci. U.S.A. 112, 4582 (2015).

[19] J. Eggers, Phys. Rev. Lett. 71, 3458 (1993).

[20] Y. J. Chen and P. H. Steen, J. Fluid Mech. 341, 245 (1997).

[21] J. Eggers, Rev. Mod. Phys. 69, 865 (1997).
[22] J. R. Lister and H. A. Stone, Phys. Fluids 10, 2758 (1998).

[23] R. F. Day, E. J. Hinch, and J. R. Lister, Phys. Rev. Lett. 80, 704 (1998).

[24] D. T. Papageorgiou, Phys. Fluids 7, 1529 (1995).

[25] J. C. Burton, J. E. Rutledge, and P. Taborek, Phys. Rev. Lett. 92, 244505 (2004).

[26] A. U. Chen, P. K. Notz, and O. A. Basaran, Phys. Rev. Lett. 88, 174501 (2002).

[27] A. Deblais, M. A. Herrada, I. Hauner, K. P. Velikov, T. van Roon, H. Kellay, J. Eggers, and D. Bonn, Phys. Rev. Lett. 121, 254501 (2018).

[28] A. Lagarde, C. Josserand, and S. Protiere, Proc. Natl. Acad. Sci. U.S.A. 115, 12371 (2018).

[29] See Supplemental Material at http://link.aps.org/ supplemental/10.1103/PhysRevLett.123.234502, which includes Refs. [30,31], for more experimental and numerical details, effect of impact angle, and the consistency between the present results and previous studies.

[30] M. Liu and D. Bothe, J. Fluid Mech. 789, 785 (2016).

[31] H. A. Stone, B. J. Bentley, and L. G. Leal, J. Fluid Mech. 173, 131 (1986).

[32] J. R. Castrejon-Pita, A. A. Castrejon-Pita, E. J. Hinch, J. R. Lister, and I. M. Hutchings, Phys. Rev. E 86, 015301(R) (2012).

[33] X. D. Shi, M. P. Brenner, and S. R Nagel, Science 265, 219 (1994).

[34] C. Gotaas, P. Havelka, H. A. Jakobsen, H. F. Svendsen, M. Hase, N. Roth, and B. Weigand, Phys. Fluids 19, 102106 (2007).

[35] K. D. Willis and M. E. Orme, Exp. Fluids 29, 347 (2000).

[36] S. Guido and M. Simeone, J. Fluid Mech. 357, 1 (1998).

[37] V. Cristini, S. Guido, A. Alfani, J. Bławzdziewicz, and M. Loewenberg, J. Rheol. 47, 1283 (2003).

[38] S. Popinet, J. Comput. Phys. 190, 572 (2003).

[39] S. Popinet, J. Comput. Phys. 228, 5838 (2009).

[40] M.-J. Thoraval, K. Takehara, T. G. Etoh, S. Popinet, P. Ray, C. Josserand, S. Zaleski, and S. T. Thoroddsen, Phys. Rev. Lett. 108, 264506 (2012).

[41] X. Chen and V. Yang, J. Comput. Phys. 269, 22 (2014).

[42] P. K. Notz and O. A. Basaran, J. Fluid Mech. 512, 223 (2004).

[43] A. Rothert, R. Richter, and I. Rehberg, Phys. Rev. Lett. 87, 084501 (2001).

[44] C. Tang, P. Zhang, and C. K. Law, Phys. Fluids 24, 022101 (2012).

[45] R. H. Chen and C. T. Chen, Exp. Fluids 41, 453 (2006).

[46] C. Planchette, H. Hinterbichler, M. Liu, D. Bothe, and G. Brenn, J. Fluid Mech. 814, 277 (2017).

[47] I. V. Roisman, C. Planchette, E. Lorenceau, and G. Brenn, J. Fluid Mech. 690, 512 (2012).

[48] J. Eggers, J. Appl. Math. Mech. 85, 400 (2005). 